Pure and Applied Mathematics Quarterly

Volume 6, Number 4

(Special Issue: In honor of

Joseph J. Kohn, Part 2 of 2$)$

1123-1143, 2010

\title{
Some Improved Caffarelli-Kohn-Nirenberg Inequalities with General Weights and Optimal Remainders*
}

\author{
Yaotian Shen and Zhihui Chen \\ Dedicated to Professor J.J.Kohn on the occasion of his 75th birthday
}

\begin{abstract}
In this paper, we establish some improved Caffarelli-KohnNirenberg inequalities with general weights and optimal remainders. Moreover, we give a positive answer to an open problem raised by Abdellaoui et al. [1].
\end{abstract}

Keywords: Hardy-Sobolev inequality, general weight, optimal remainders

\section{Introduction}

Let $p>1$ be a constant. In 1920, Hardy [7] showed that, for any positive $f(x) \in L^{p}(0, \infty)$,

$$
\int_{0}^{\infty}\left[\frac{F(x)}{x}\right]^{p} \mathrm{~d} x \leq\left(\frac{p}{p-1}\right)^{p} \int_{0}^{\infty}|f(x)|^{p} \mathrm{~d} x,
$$

where $F(x)=\int_{0}^{x} f(t) \mathrm{d} t$, and the constant $\left(\frac{p}{p-1}\right)^{p}$ is optimal.

Received February 6, 2008.

2000 MR Subject Classification, 46E35, 35J40

*Project supported by the National Natural Science Foundation of China (No. 10771074, 10726060) and the Natural Science Foundation of Guangdong Province (No. 04020077) 
In 1933, Leray [8] gave the following multidimensional version of Hardy's inequality

$$
\begin{array}{r}
\int_{\mathbb{R}^{2} \backslash B_{1}(0)} \frac{u^{2}}{|x|^{2} \ln ^{2}|x|} \mathrm{d} x \leq 4 \int_{\mathbb{R}^{2} \backslash B_{1}(0)}|\nabla u|^{2} \mathrm{~d} x, \quad u \in C_{0}^{\infty}\left(\mathbb{R}^{2} \backslash B_{1}(0)\right) \\
\int_{\mathbb{R}^{N}} \frac{u^{2}}{|x|^{2}} \mathrm{~d} x \leq\left(\frac{2}{N-2}\right)^{2} \int_{\mathbb{R}^{N}}|\nabla u|^{2} \mathrm{~d} x, \quad u \in C_{0}^{\infty}\left(\mathbb{R}^{N}\right), \quad N \geq 3
\end{array}
$$

We may call the above two inequalities Hardy-Leray inequality, which is called Hardy-Sobolev inequality in the literature (see [1]). For any bounded domain $\Omega \subset B_{R}(0)$ including origin, $B_{R}(0)$ denotes a ball in $\mathbb{R}^{N}$ with radius $R$ and centered at 0 , Shen [9] obtained (1.1) with $\ln ^{2}|x|$ being replaced by $\ln ^{2} R /|x|$.

Brézis and Vázques [3] obtained a remainder term for the Hardy-Leray's inequality. More precisely, if $1 \leq q<\frac{2 N}{N-2}, N \geq 3$, there exists a constant $C(q,|\Omega|)>0$ such that

$$
\int_{\Omega}|\nabla u|^{2} \mathrm{~d} x-\frac{(N-2)^{2}}{4} \int_{\Omega} \frac{u^{2}}{|x|^{2}} \mathrm{~d} x \geq C(q,|\Omega|)\left(\int_{\Omega}|u|^{q} \mathrm{~d} x\right)^{2 / q}, \quad u \in H_{0}^{1}(\Omega)
$$

They raised some open problems in [3], and the second one states whether there is a further improvement in the direction of this inequality.

Vázquez and Zuazua [16], among other results, improved the previous inequality by showing that if $1<q<2$, there exists a constant $C(q,|\Omega|)>0$ such that, for each $u \in H_{0}^{1}(\Omega)$,

$$
\int_{\Omega}|\nabla u|^{2} \mathrm{~d} x-\frac{(N-2)^{2}}{4} \int_{\Omega} \frac{u^{2}}{|x|^{2}} \mathrm{~d} x \geq C(q,|\Omega|)\left(\int_{\Omega}|\nabla u|^{q} \mathrm{~d} x\right)^{2 / q}
$$

The Caffarelli-Kohn-Nirenberg inequality [4] shows that, if $1<p<N$ and $\gamma<\frac{N-p}{p}$, for any $u \in C_{0}^{\infty}(\Omega)$,

$$
c_{p} \int_{\Omega}|x|^{-p(\gamma+1)}|u|^{p} \mathrm{~d} x \leq \int_{\Omega}|x|^{-p \gamma}|\nabla u|^{p} \mathrm{~d} x
$$

where $\Omega$ is allowed to be the whole space $\mathbb{R}^{N}$.

Wang and Willem [17] obtained the Caffarelli-Kohn-Nirenberg inequality with 
optimal remainder, that is, if $0 \in \Omega \subset B_{R}(0)$, then for any $u \in H_{0}^{1}(\Omega)$,

$$
\begin{aligned}
\int_{\Omega}|x|^{-2 \gamma}|\nabla u|^{2} \mathrm{~d} x-\left[\frac{N-2(\gamma+1)}{2}\right]^{2} & \int_{\Omega}|x|^{-2(\gamma+1)}|u|^{2} \mathrm{~d} x \\
& \geq C \int_{\Omega}|x|^{-2 \gamma}(\ln R /|x|)^{-2}|\nabla u|^{2} \mathrm{~d} x
\end{aligned}
$$

It is optimal in the sense that $(\ln R /|x|)^{-1}$ can not be replaced by $g(x)(\ln R /|x|)^{-1}$ with $g$ satisfying $|g(x)| \rightarrow \infty$ as $|x| \rightarrow 0$. If $\gamma=0,(1.6)$ gives a positive answer to the second open problem of [3] in some sense. The authors proved another result which works for bounded domains as well as exterior domains, that is,

$$
\begin{aligned}
\int_{\Omega}|x|^{-2 \gamma}|\nabla u|^{2} \mathrm{~d} x-\left[\frac{N-2(\gamma+1)}{2}\right]^{2} \int_{\Omega}|x|^{-2(\gamma+1)}|u|^{2} \mathrm{~d} x & \\
& \geq \frac{1}{4} \int_{\Omega}|x|^{-2(\gamma+1)}(\ln R /|x|)^{-2} u^{2} \mathrm{~d} x,
\end{aligned}
$$

where $\gamma \leq \frac{N-p}{p}, \Omega \subset B_{R}(0)$ or $\Omega \subset B_{R}^{C}(0)$. Moreover, the constant $\frac{1}{4}$ is also sharp.

Abdellaoui et al. [1] proved that if $1<q<p<N$, then for any $u \in C_{0}^{\infty}(\Omega)$,

$$
\int_{\Omega}|x|^{-\gamma p}|\nabla u|^{p} \mathrm{~d} x-\left[\frac{N-p(\gamma+1)}{p}\right]^{p} \int_{\Omega} \frac{|u|^{p}}{|x|^{p(\gamma+1)}} \mathrm{d} x \geq C \int_{\Omega}|x|^{-\gamma r}|\nabla u|^{q} \mathrm{~d} x
$$

where $q<r<+\infty$ if $\gamma \leq 0$, or $r<p+\rho(N, p, q, \gamma)$ for some positive constant $\rho$ if $\gamma>0$. The authors point out that it seems to be an open problem to obtain the best weight for (1.7) as in (1.6), in the case $p \neq 2$. In this paper, we give a positive answer to this open problem. In fact, we obtain the CaffarelliKohn-Nirenberg inequality with general weights and remainder term. Because the weight is general, we also obtain the corresponding inequality with weight $|x|^{-\gamma p}$ in the case of $N=p>1$. When $N=p=2$, this problem has been discussed in [14].

Now we introduce the weighted Sobolev space. Let $\phi$ be a positive continuous function with $\phi(|x|) \in L\left(B_{\delta}(0)\right)$ for some positive $\delta$, and define

$$
\bar{h}\left(r_{1}, r_{2}\right)=c_{0} \int_{r_{1}}^{r_{2}}\left(\phi r^{N-1}\right)^{-1 /(p-1)} \mathrm{d} r
$$

for $0 \leq r_{1} \leq r_{2} \leq \infty$, where $c_{0}$ is a given positive constant. In this paper, we consider the following two cases: 
$\left(\mathrm{A}_{1}\right) \bar{h}(r, \infty)<\infty$ for all $r>0$ and $\bar{h}(0, \infty)=\infty$;

$\left(\mathrm{A}_{2}\right) \bar{h}(r, \infty)=\infty$ and $\bar{h}(0, D)=\infty$ for some $r, D>0$.

Definition 1. Let $p>1$, we denote by $W_{0}^{1, p}(\Omega, \phi)$ the completion of $C_{0}^{\infty}(\Omega)$ with respect to the norm

$$
\|u\|_{1, p, \phi}=\left(\int_{\Omega} \phi(r)|\nabla u|^{p} \mathrm{~d} x\right)^{1 / p}
$$

where $r=|x|$.

Example 1. Let $\phi=r^{-p \gamma}$ and $0 \in \Omega \subset B_{D}(0)$. If $\gamma<\frac{N-p}{p}$, then $\left(\mathrm{A}_{1}\right)$ happens, and $W_{0}^{1, p}\left(\Omega,|x|^{-p \gamma}\right)$ is identical with $D_{0, \gamma}^{1, p}(\Omega)$ in [1]. If $\gamma=\frac{N-p}{p}$, then $\left(\mathrm{A}_{2}\right)$ happens, and $W_{0}^{1, p}\left(\Omega,|x|^{-p \gamma}\right)$ has not been discussed before.

In what follows, for short, we use $\phi$ for $\phi(r)$ or $\phi(|x|)$, etc.

Set

$$
\bar{h}= \begin{cases}\bar{h}(r, \infty), & \text { if }\left(\mathrm{A}_{1}\right) \text { holds } \\ \bar{h}(r, D), & \text { if }\left(\mathrm{A}_{2}\right) \text { holds }\end{cases}
$$

If $N>p$ and $\phi \equiv 1$, then $\left(\mathrm{A}_{1}\right)$ holds, therefore $\bar{h}(|x|)=|x|^{\frac{p-N}{p-1}}$ is a fundamental solution for the $p$-Laplace operator. For general weight $\phi$, function $h=\bar{h}^{(p-1) / p}$ satisfies in the sense of distribution

$$
-\Delta_{\phi, p} u=: \operatorname{div}\left(\phi|\nabla u|^{p-2} \nabla u\right)=\psi|u|^{p-2} u
$$

where $\psi=\left(\frac{p-1}{p}\right)^{p} \phi\left(-\frac{\bar{h}^{\prime}}{h}\right)^{p}=\phi\left(-\frac{h^{\prime}}{h}\right)^{p}$, that is, $h$ is a weak solution of the Euler-Lagrange equation (1.8) of the functional

$$
I_{1, \phi}(u)=: \int_{\Omega}\left(\phi|\nabla u|^{p}-\psi|u|^{p}\right) \mathrm{d} x
$$

In [10][11][12] it has been proved that if $\phi, \psi$ are positive functions in $C^{1}(0, a)$ and satisfy the Bernoulli equation

$$
\left(\phi^{1 / p} \psi^{1-1 / p}\right)^{\prime}+\frac{N-1}{r} \phi^{1 / p} \psi^{1-1 / p}=p \psi
$$

then for any $u \in C_{0}^{\infty}(\Omega)$,

$$
\int_{\Omega} \psi|u|^{p} \mathrm{~d} x \leq \int_{\Omega} \phi|\nabla u|^{p} \mathrm{~d} x
$$


and the constant 1 is optimal, where $a=+\infty$ and $\Omega=\mathbb{R}^{N}$ if $\left(\mathrm{A}_{1}\right)$ holds, or $a=D$ and $\Omega \subset B_{D}(0)$ if $\left(\mathrm{A}_{2}\right)$ holds. Because $\bar{h}$ is a fundamental solution of operator $-\Delta_{p, \phi}$, in other words, $h$ is a distribution solution of equation (1.8), we know $\psi$ can be expressed by $\bar{h}$ and $\phi$ or by $h$ and $\phi$ as follows

$$
\psi=\left(\frac{p-1}{p}\right)^{p} \phi\left(-\frac{\bar{h}^{\prime}}{\bar{h}}\right)^{p}=\phi\left(-\frac{h^{\prime}}{h}\right)^{p}
$$

Theorem 1.1 ([5], Theorem 1.1). Let $\Omega$ be $\mathbb{R}^{N}$ if $\left(A_{1}\right)$ holds or $\Omega$ be a bounded domain included in $B_{D}(0)$ if $\left(A_{2}\right)$ holds. Suppose that $\phi$ is continuous and set

$$
h=\left(c_{0} \int_{r}^{a}\left(\phi r^{N-1}\right)^{-1 /(p-1)} \mathrm{d} r\right)^{(p-1) / p}
$$

where $a=+\infty$ if $\left(A_{1}\right)$ holds or $a=D$ if $\left(A_{2}\right)$ holds. Then for any $u \in W_{0}^{1, p}(\Omega, \phi)$

$$
\int_{\Omega} \phi\left(-\frac{h^{\prime}}{h}\right)^{p}|u|^{p} \mathrm{~d} x \leq \int_{\Omega} \phi|\nabla u|^{p} \mathrm{~d} x
$$

where the constant 1 is optimal.

Remark 1.1. $\left(\mathrm{A}_{1}\right)$ or $\left(\mathrm{A}_{2}\right)$ implies the integrability of $\phi\left(-\frac{h^{\prime}}{h}\right)^{p}$ in $B_{\delta}(0)$.

Theorem 1.2. Let $p>1$ and $\Omega$ be a bounded domain in $\mathbb{R}^{N}$. Suppose $\phi$ is continuous satisfying $\left(A_{1}\right)$ or $\left(A_{2}\right), h$ is defined by (1.11). Set

$$
h_{1}= \begin{cases}\frac{p}{(p-1) c_{0}} \ln \frac{h(r)}{h(D)}, & \text { if }\left(A_{1}\right) \text { holds } \\ \frac{p}{(p-1) c_{0}} \ln h(r), & \text { if }\left(A_{2}\right) \text { holds }\end{cases}
$$

then

(1) There exists a positive constant $D_{0} \leq D$ such that for any $u \in W_{0}^{1, p}(\Omega, \phi)$

$$
\int_{\Omega} \phi|\nabla u|^{p} \mathrm{~d} x-\int_{\Omega} \psi|u|^{p} \mathrm{~d} x \geq \frac{p}{2(p-1) c_{0}^{2}} \int_{\Omega} \psi h_{1}^{-2}|u|^{p} \mathrm{~d} x
$$

where $\psi=\phi\left(-\frac{h^{\prime}}{h}\right)^{p}$.

(2) The constants in (1.13) are optimal, that is,

$$
\frac{p}{2(p-1) c_{0}^{2}}=\inf _{W_{0}^{1, p}(\Omega, \phi)} \frac{I_{\phi}(u)}{\int_{\Omega} \psi h_{1}^{-2}|u|^{p} \mathrm{~d} x}
$$


Remark 1.2. Let $\phi=r^{-p \gamma}$ with $\gamma<\frac{N-p}{p}$ and $c_{0}=\frac{N-p(\gamma+1)}{p-1}$. It follows from (1.11) and (1.12) that

$$
h=r^{-\frac{N-p(\gamma+1)}{p}}, \quad \psi=\left(\frac{N-p(\gamma+1)}{p}\right)^{p} r^{-p(\gamma+1)}, \quad h_{1}=\ln \frac{D}{r}
$$

hence we obtain by (1.13)

$$
\begin{aligned}
\int_{\Omega}\left(|x|^{-p \gamma}|\nabla u|^{p}\right. & \left.-\left(\frac{N-p(\gamma+1)}{p-1}\right)^{p}|x|^{-p(\gamma+1)}|u|^{p}\right) \mathrm{d} x \\
& \geq \frac{p-1}{2 p}\left(\frac{N-p(\gamma+1)}{p-1}\right)^{p-2} \int_{\Omega}|x|^{-p(\gamma+1)}(\ln D /|x|)^{-2}|u|^{p} \mathrm{~d} x
\end{aligned}
$$

which is identical with Theorem A in [2] when $\gamma=0$.

Remark 1.3. Theorem 1.2 improves the results of [13][15].

Theorem 1.3. Under the hypothesis of Theorem 1.2, we have

i)

$$
\int_{\Omega} \phi|\nabla u|^{p}-\psi|u|^{p} \mathrm{~d} x \geq C \int_{\Omega} \phi h_{1}^{-2}|\nabla u|^{p} \mathrm{~d} x, \quad \forall u \in W_{0}^{1, p}(\Omega, \phi)
$$

ii) The inequality (1.14) is optimal in the sense that $h_{1}^{-2}$ can not be replaced by any weight of the form $g(x) h_{1}^{-2}$ where $g(x)$ is a positive function such that $g(x) \rightarrow \infty$ as $x \rightarrow 0$.

Remark 1.4. Taking $\phi=r^{-p \gamma}$ in Theorem 1.3, if $\gamma<\frac{N-p}{p}$, then

$$
\begin{aligned}
\int_{\Omega}\left(|x|^{-p \gamma}|\nabla u|^{p}-\left(\frac{N-p(\gamma+1)}{p-1}\right)^{p}|x|^{-p(\gamma+1)}|u|^{p}\right) \mathrm{d} x & \\
& \geq C \int_{\Omega}|x|^{-p \gamma}(\ln D /|x|)^{-2}|\nabla u|^{p} \mathrm{~d} x
\end{aligned}
$$

for any $u \in W_{0}^{1, p}(\Omega, \phi)$. This is a positive answer to the open problem in [1]. If $\gamma=\frac{N-p}{p}$, then

$$
\begin{aligned}
\int_{\Omega}\left(|x|^{-p \gamma}|\nabla u|^{p}-\left(\frac{p-1}{p}\right)^{p}|x|^{-p(\gamma+1)}\left(\ln D^{\prime} /|x|\right)^{-p}|u|^{p}\right) \mathrm{d} x \\
\quad \geq C \int_{\Omega}|x|^{-p(\gamma+1)}\left(\ln D^{\prime} /|x|\right)^{-p}\left(\ln \ln D^{\prime} /|x|\right)^{-2}|\nabla u|^{p} \mathrm{~d} x
\end{aligned}
$$

for any $u \in W_{0}^{1, p}(\Omega, \phi)$, where $D^{\prime}>\mathrm{e} D$. This solves the problem for the case of $\gamma=\frac{N-p}{p}$ which has not been discussed before. 
Remark 1.5. Wang and Willem [17] proved (1.6) by using a change of variable that appear in [6]. However, to prove Theorem 1.3, we use a change of variables that appear in [14] $(p=2)$, which involves the function $\bar{h}$ or the distribution solution $h$.

Remark 1.6. Theorem 1.3 gives a positive answer to the second open problem of $[3]$ in the case of general weights.

\section{Some Lemmas and Corollaries}

Lemma 2.1 ([1]). For all $\zeta_{1}, \zeta_{2} \in \mathbb{R}^{N}$, the following inequalities hold

i) if $p \leq 2$,

$$
\left|\zeta_{2}\right|^{p}-\left|\zeta_{1}\right|^{p}-p\left|\zeta_{1}\right|^{p-2}\left\langle\zeta_{1}, \zeta_{2}-\zeta_{1}\right\rangle \geq c(p) \frac{\left|\zeta_{2}-\zeta_{1}\right|^{2}}{\left(\left|\zeta_{1}\right|+\left|\zeta_{2}\right|\right)^{2-p}}
$$

ii) if $p>2$,

$$
\left|\zeta_{2}\right|^{p}-\left|\zeta_{1}\right|^{p}-p\left|\zeta_{1}\right|^{p-2}\left\langle\zeta_{1}, \zeta_{2}-\zeta_{1}\right\rangle \geq c(p)\left|\zeta_{2}-\zeta_{1}\right|^{p}
$$

Direct calculations give the following results:

Lemma 2.2. Assume $h$ satisfies (1.11). If $\left(A_{1}\right)$ or $\left(A_{2}\right)$ happens, then

$$
\operatorname{div}\left(\phi h^{\alpha}\left(-h^{\prime}\right)^{p-1} \frac{x}{|x|}\right)=(1-\alpha) \phi h^{\alpha-1}\left(-h^{\prime}\right)^{p}
$$

Lemma 2.3. Let $h=\left(c_{0} \int_{r}^{\infty}\left(\phi r^{N-1}\right)^{-1 /(p-1)} \mathrm{d} r\right)^{(p-1) / p}$. Then

i) the function $h$ satisfies the Euler-Lagrange equation

$$
-\operatorname{div}\left(\phi|\nabla h|^{p-2} \nabla h\right)=\psi h^{p-1}, \quad x \in \mathbb{R}^{N} \backslash\{0\}
$$

and in weak sense,

$$
\int_{\mathbb{R}^{N}} \phi|\nabla h|^{p-2} \nabla h \nabla \zeta \mathrm{d} x=\int_{\mathbb{R}^{N}} \psi h^{p-1} \zeta \mathrm{d} x, \quad \zeta \in C_{0}^{\infty}\left(\mathbb{R}^{N}\right)
$$

where $\psi=\phi\left(-\frac{h^{\prime}}{h}\right)^{p}$; 
ii) the function

$$
\bar{h}=h^{p /(p-1)}=c_{0} \int_{r}^{\infty}\left(\phi r^{N-1}\right)^{-1 /(p-1)} \mathrm{d} r
$$

satisfies in the sense of distribution

$$
-\operatorname{div}\left(\phi|\nabla \bar{h}|^{p-2} \nabla \bar{h}\right)=\left(\frac{p}{p-1}\right)^{p-1} \omega_{N} \delta(x)
$$

where $\delta(x)$ is the Dirac measure and $\omega_{N}$ denotes the volume of the unit ball in $\mathbb{R}^{N}$. In other words, $\bar{h}$ is a fundamental solution for operator $-\Delta_{\phi, p}$ defined as before.

Corollary 2.4. Under the hypothesis of Theorem 1.2, if $\alpha>0$, then for any $u \in W_{0}^{1, p}(\Omega, \phi)$,

$$
\int_{\Omega} \psi h_{1}^{-\alpha}|u|^{p} \mathrm{~d} x \leq \int_{\Omega} \phi h_{1}^{-\alpha}|\nabla u|^{p} \mathrm{~d} x
$$

Proof. Assume $\left(\mathrm{A}_{1}\right)$ holds. Set $\bar{\phi}=\phi h_{1}^{-\alpha}$, then

$$
-\frac{\bar{h}^{\prime}}{\bar{h}}=\frac{p-1}{p} \frac{\left(\phi h_{1}^{-\alpha} r^{N-1}\right)^{-1 /(p-1)}}{\int_{r}^{D}\left(\phi h_{1}^{-\alpha} r^{N-1}\right)^{-1 /(p-1)} \mathrm{d} r}
$$

By Theorem 1.1, we have

$$
\int_{\Omega} \bar{\psi}|u|^{p} \mathrm{~d} x \leq \int_{\Omega} \bar{\phi}|\nabla u|^{p} \mathrm{~d} x
$$

where $\bar{\psi}=\bar{\phi}\left(-\frac{h^{\prime}}{h}\right)^{p}$. We claim that

$$
\psi h_{1}^{-\alpha} \leq \bar{\psi}
$$

that is

$$
\phi h_{1}^{-\alpha}\left(-\frac{h^{\prime}}{h}\right)^{p} \leq \phi h_{1}^{-\alpha}\left(-\frac{\bar{h}^{\prime}}{\bar{h}}\right)^{p}
$$

and this complete the proof. In the following we prove this claim. Since $h_{1}$ is decreasing, we have

$$
\int_{r}^{D}\left(\phi h_{1}^{-\alpha} r^{N-1}\right)^{-1 /(p-1)} \mathrm{d} r \leq h_{1}^{\alpha /(p-1)} \int_{r}^{D}\left(\phi r^{N-1}\right)^{-1 /(p-1)} \mathrm{d} r
$$

Multiplying by $\left(\phi r^{N-1}\right)^{-1 /(p-1)}$, we obtain

$$
\frac{\left(\phi r^{N-1}\right)^{-1 /(p-1)}}{\int_{r}^{D}\left(\phi r^{N-1}\right)^{-1 /(p-1)} \mathrm{d} r} \leq \frac{\left(\phi h_{1}^{-\alpha} r^{N-1}\right)^{-1 /(p-1)}}{\int_{r}^{D}\left(\phi h_{1}^{-\alpha} r^{N-1}\right)^{-1 /(p-1)} \mathrm{d} r}
$$


Hence

that is, the claim is true.

$$
-\frac{h^{\prime}}{h} \leq-\frac{\bar{h}^{\prime}}{\bar{h}}
$$

\section{Proof of Theorem}

Proof of Theorem 1.2 (1). We proceed to make use of a suitable vector field as in [2]. Define a vector field as follows

$$
T=\phi\left(-\frac{h^{\prime}}{h}\right)^{p-1}\left(1+c_{0}^{-1} \eta+a \eta^{2}\right) \nabla r
$$

where $a$ is a free parameter to be chosen later and $\eta=h_{1}^{-1}$. By Lemma 2.1, we have

$$
\operatorname{div} T \geq \phi\left(-\frac{h^{\prime}}{h}\right)^{p}\left[\left(p+p c_{0}^{-1} \eta+a p \eta^{2}\right)+\frac{p \eta^{2}}{(p-1) c_{0}^{2}}+\frac{2 a p \eta^{3}}{(p-1) c_{0}}\right]
$$

Next we compute $(p-1) \phi^{-1 /(p-1)}|T|^{p /(p-1)}$. We set for convenience

$$
g(\eta)=\left(1+c_{0}^{-1} \eta+a \eta^{2}\right)^{p /(p-1)}
$$

When $\eta>0$ is small, the Taylor expansion of $g(\eta)$ about $\eta=0$ gives

$$
\begin{aligned}
g(\eta)=1+\frac{p}{(p-1) c_{0}} \eta+\frac{1}{2}\left(\frac{p}{(p-1)^{2} c_{0}^{2}}+\frac{2 p a}{p-1}\right) \eta^{2} \\
\quad+\frac{1}{6}\left(\frac{p(2-p)}{(p-1)^{3} c_{0}^{3}}+\frac{6 p a}{(p-1)^{2} c_{0}}\right) \eta^{3}+O\left(\eta^{4}\right)
\end{aligned}
$$

and so

$$
\begin{aligned}
& (p-1) \phi^{-1 /(p-1)}|T|^{p /(p-1)}=\phi\left(-\frac{h^{\prime}}{h}\right)^{p}\left[(p-1)+\frac{p}{c_{0}} \eta\right. \\
& \left.+\left(\frac{p}{2(p-1) c_{0}^{2}}+p a\right) \eta^{2}+\left(\frac{p(2-p)}{(p-1)^{2} c_{0}^{3}}+\frac{p a}{(p-1) c_{0}}\right) \eta^{3}+O\left(\eta^{4}\right)\right]
\end{aligned}
$$

Hence

$$
\begin{aligned}
& \operatorname{div} T-(p-1) \phi^{-1 /(p-1)}|T|^{p /(p-1)} \\
\geq & \phi\left(-\frac{h^{\prime}}{h}\right)^{p}\left[1+\frac{p \eta^{2}}{2(p-1) c_{0}^{2}}+\left(\frac{p a}{(p-1) c_{0}}-\frac{p(2-p)}{(p-1)^{2} c_{0}^{3}}\right) \eta^{3}+O\left(\eta^{4}\right)\right]
\end{aligned}
$$


If we show

$$
\frac{a p}{(p-1) c_{0}} \geq \frac{p(2-p)}{(p-1)^{2} c_{0}^{3}}+O(\eta)
$$

then we obtain

$$
\operatorname{div} T-(p-1) \phi^{-1 /(p-1)}|T|^{p /(p-1)} \geq \phi\left(-\frac{h^{\prime}}{h}\right)^{p}\left[1+\frac{p \eta^{2}}{2(p-1) c_{0}^{2}}\right]
$$

If $1<p<2$, we assume that $\eta$ is small for the case $\left(\mathrm{A}_{1}\right)$. Since

$$
h_{1}=\frac{p}{(p-1) c_{0}} \ln \frac{h(r)}{h(D)}
$$

and $\Omega \subset B_{D_{0}}(0)$ is bounded, we can choose $D_{0}$ large enough such that $h_{1}^{-1}\left(D_{0}\right)$ is small enough. Then $\eta=h_{1}^{-1}$ is small. Hence, we have (3.2) for $a$ big enough. The same argument gives (3.2) for the case $\left(\mathrm{A}_{2}\right)$.

If $p \geq 2$, we choose $a=0$, then

$$
\left(1+c_{0}^{-1} \eta\right)^{\frac{p}{p-1}}=1+\frac{p}{(p-1) c_{0}} \eta+\frac{p}{2(p-1)^{2} c_{0}^{2}} \eta^{2}+\frac{p(2-p)}{6(p-1)^{3} c_{0}^{3}}\left(1+c_{0}^{-1} \xi\right)^{\frac{3-2 p}{p-1}} \eta^{3}
$$

for some $\xi \in(0, \eta)$, without any smallness assumption. Since $2-p \leq 0$, we have

$$
\left(1+c_{0}^{-1} \eta\right)^{\frac{p}{p-1}} \leq 1+\frac{p}{(p-1) c_{0}} \eta+\frac{p}{2(p-1)^{2} c_{0}^{2}} \eta^{2}
$$

Hence we prove (3.3).

Let $u \in C_{0}^{\infty}(\Omega)$. For $\epsilon>0$, it follows from integration by parts that

$$
\int_{\Omega \backslash B_{\epsilon}(0)}|u|^{p} \operatorname{div} T \mathrm{~d} x=-p \int_{\Omega \backslash B_{\epsilon}(0)}(T \cdot \nabla u)|u|^{p-2} u \mathrm{~d} x-\int_{\partial B_{\epsilon}(0)}|u|^{p} T \cdot \nabla r \mathrm{~d} S
$$

Note that

$$
\phi\left(-\frac{h^{\prime}}{h}\right)^{p-1}=r^{-(N-1)}\left(\int_{r}^{a}\left(\phi r^{N-1}\right)^{-1 /(p-1)} \mathrm{d} r\right)^{-(p-1)}=r^{-(N-1)} h^{-p /(p-1)^{2}}(r)
$$

then

$$
\left.\left.\left|\int_{\partial B_{\epsilon}(0)}\right| u\right|^{p} T \cdot \nabla r \mathrm{~d} S\left|\leq \int_{\partial B_{\epsilon}(0)}\right| u\right|^{p} \epsilon^{-(N-1)} h^{-p /(p-1)^{2}}(\epsilon) \mathrm{d} S
$$

which tends to 0 as $\epsilon \rightarrow 0$ since $h^{-1}(0)=0$. Hence we obtain

$$
\int_{\Omega}|u|^{p} \operatorname{div} T \mathrm{~d} x=-p \int_{\Omega}(T \cdot \nabla u)|u|^{p-2} u \mathrm{~d} x
$$


By Hölder's inequality and Young's inequality, we have

$$
\begin{aligned}
\int_{\Omega}|u|^{p} \operatorname{div} T \mathrm{~d} x & \leq p\left(\int_{\Omega} \phi|\nabla u|^{p} \mathrm{~d} x\right)^{1 / p}\left(\int_{\Omega}\left|T \phi^{-1 / p}\right|^{p /(p-1)}|u|^{p} \mathrm{~d} x\right)^{(p-1) / p} \\
& \leq \int_{\Omega} \phi|\nabla u|^{p} \mathrm{~d} x+(p-1) \int_{\Omega}\left|T \phi^{-1 / p}\right|^{p /(p-1)}|u|^{p} \mathrm{~d} x
\end{aligned}
$$

that is,

$$
\int_{\Omega} \phi|\nabla u|^{p} \mathrm{~d} x \geq \int_{\Omega}\left(\operatorname{div} T-(p-1)\left|T \phi^{-1 / p}\right|^{p /(p-1)}\right)|u|^{p} \mathrm{~d} x
$$

This complete the proof by (3.3).

Proof of Theorem 1.2 (2). We complete the proof by four steps.

Step 1. Let $\theta \in C_{0}^{\infty}\left(B_{\delta}\right)$ be such that $0 \leq \theta \leq 1$ in $B_{\delta}$ and $\theta=1$ in $B_{\delta / 2}$, where $B_{\delta}$ denotes the ball of radius $\delta$ centered at the origin. We fix small positive parameters $\alpha_{0}, \alpha_{1}$ and define the functions

$$
w(x)=h^{1-\frac{\alpha_{0}}{(p-1) c_{0}}} h_{1}^{\frac{1-\alpha_{1}}{p}}
$$

and

$$
u(x)=\theta(x) w(x)
$$

Let $\left(\mathrm{A}_{1}\right)$ or $\left(\mathrm{A}_{2}\right)$ happen. Hence $u \in W_{0}^{1, p}(\Omega, \phi)$. To prove the proposition we shall estimate the corresponding Rayleigh quotient of $u$ in the limit of the order $\alpha_{0} \rightarrow 0, \alpha_{1} \rightarrow 0$.

It is easily seen that

$$
\nabla w=\frac{p}{(p-1) c_{0}} h^{-\frac{\alpha_{0}}{(p-1) c_{0}}} h^{\prime} Y_{1}^{\frac{-1+\alpha_{1}}{p}}\left(\frac{(p-1) c_{0}}{p}+\frac{\eta}{p}\right) \nabla r
$$

where $Y_{1}=h_{1}^{-1}$ and $\eta=-\alpha_{0}+\left(1-\alpha_{1}\right) Y_{1}$.

Now $\nabla u=\theta \nabla w+w \nabla \theta$ and hence, using the elementary inequality

$$
|a+b|^{p} \leq|a|^{p}+c_{p}\left(|a|^{p-1}|b|+|b|^{p}\right), \quad a, b \in \mathbb{R}^{N}
$$


for $p>1$, we obtain

$$
\begin{aligned}
\int_{\Omega} \phi|\nabla u|^{p} \mathrm{~d} x & \leq \int_{\Omega} \phi \theta^{p}|\nabla w|^{p} \mathrm{~d} x \\
& +c_{p} \int_{\Omega} \phi \theta^{p-1}|\nabla \theta||w||\nabla w|^{p-1} \mathrm{~d} x+c_{p} \int_{\Omega} \phi|\nabla \theta|^{p}|w|^{p} \mathrm{~d} x \\
& =: I_{1}+I_{2}+I_{3}
\end{aligned}
$$

We claim that

$$
I_{2}, I_{3}=O(1) \text { uniformly as } \alpha_{0}, \alpha_{1} \text { tend to zero. }
$$

Let us give the proof for $I_{2}$. In fact,

$$
\begin{aligned}
& I_{2} \leq C \int_{B_{\delta}} \phi h^{-\frac{\alpha}{c_{0}}}\left|h^{\prime}\right|^{p-1} Y_{1}^{\frac{\left(-1+\alpha_{1}\right)(p-1)}{p}}\left[(p-1) c_{0}+\alpha_{0}+\left(1-\alpha_{1}\right) Y_{1}\right]^{p-1} \\
& \cdot h^{1-\frac{\alpha_{0}}{(p-1) c_{0}}} Y_{1}^{\frac{-1+\alpha_{1}}{p}} \mathrm{~d} x \\
& \quad \leq C \int_{B_{\delta}} \phi h^{1-\frac{\alpha_{0} p}{(p-1) c_{0}}}\left|h^{\prime}\right|^{p-1} Y_{1}^{-1+\alpha_{1}}\left[(p-1) c_{0}+\alpha_{0}+\left(1-\alpha_{1}\right) Y_{1}\right]^{p-1} \mathrm{~d} x
\end{aligned}
$$

It follows from the definition of $h(1.11)$ that

$$
\phi\left|h^{\prime}\right|^{p-1} h=C r^{1-N}
$$

hence

$$
I_{2} \leq C \int_{B_{\delta}} r^{1-N} h^{-\frac{\alpha_{0} p}{(p-1) c_{0}}} Y_{1}^{-1+\alpha_{1}}\left[(p-1) c_{0}+\alpha_{0}+\left(1-\alpha_{1}\right) Y_{1}\right]^{p-1} \mathrm{~d} x
$$

Then the boundedness of $h^{-1}$ together with the fact $Y_{1}(0)=0$ implies that $I_{2}$ is uniformly bounded. The integral $I_{3}$ is treated similarly.

Step 2. Define

$$
\begin{aligned}
A_{0} & =\int_{\Omega} \theta^{p} \phi h^{-\frac{\alpha_{0} p}{(p-1) c_{0}}}\left(-h^{\prime}\right)^{p} Y_{1}^{-1+\alpha_{1}} \mathrm{~d} x \\
A_{1} & =\int_{\Omega} \theta^{p} \phi h^{-\frac{\alpha_{0} p}{(p-1) c_{0}}}\left(-h^{\prime}\right)^{p} Y_{1}^{1+\alpha_{1}} \mathrm{~d} x \\
\Gamma_{01} & =\int_{\Omega} \theta^{p} \phi h^{-\frac{\alpha_{0} p}{(p-1) c_{0}}}\left(-h^{\prime}\right)^{p} Y_{1}^{\alpha_{1}} \mathrm{~d} x
\end{aligned}
$$


By Lemma 2.1, we have

$$
\phi h^{-\frac{\alpha_{0} p}{(p-1) c_{0}}}\left(-h^{\prime}\right)^{p}=\frac{(p-1) c_{0}}{p \alpha_{0}} \operatorname{div}\left(\phi h^{1-\frac{\alpha_{0} p}{(p-1) c_{0}}}\left(-h^{\prime}\right)^{p-1} \nabla r\right)
$$

Multiplying the above equality by $\theta^{p} Y_{1}^{-1+\alpha_{1}}$ and integrating over $\Omega$, we obtain

$$
\begin{aligned}
A_{0} & =\frac{(p-1) c_{0}}{p \alpha_{0}} \int_{\Omega} \theta^{p} Y_{1}^{-1+\alpha_{1}} \operatorname{div}\left(\phi h^{1-\frac{\alpha_{0} p}{(p-1) c_{0}}}\left(-h^{\prime}\right)^{p-1} \nabla r\right) \mathrm{d} x \\
& =\frac{(p-1) c_{0}}{p \alpha_{0}} \int_{\Omega} \phi h^{1-\frac{\alpha_{0} p}{(p-1) c_{0}}}\left(-h^{\prime}\right)^{p-1} \nabla\left(\theta^{p} Y_{1}^{-1+\alpha_{1}}\right) \mathrm{d} x \\
& =\frac{(p-1) c_{0}}{p \alpha_{0}}\left(-\frac{p\left(1-\alpha_{1}\right)}{(p-1) c_{0}} \int_{\Omega} \theta^{p} \phi h^{1-\frac{\alpha_{0} p}{(p-1) c_{0}}}\left(-h^{\prime}\right)^{p} Y_{1}^{\alpha_{1}} \mathrm{~d} x\right. \\
& \left.+\int_{\Omega}\left(\theta^{p}\right)^{\prime} \phi h^{1-\frac{\alpha_{0} p}{(p-1) c_{0}}}\left(-h^{\prime}\right)^{p-1} Y_{1}^{-1+\alpha} \mathrm{d} x\right) \\
& =\left(1-\alpha_{1}\right) \Gamma_{01}+O(1)
\end{aligned}
$$

Step 3. We proceed to estimate $I_{1}$.

$$
\begin{aligned}
I_{1} & =\int_{\Omega} \phi \theta^{p}|\nabla w|^{p} \mathrm{~d} x \\
& \leq\left(\frac{p}{(p-1) c_{0}}\right)^{p} \int_{\Omega} \theta^{p} \phi h^{-\frac{\alpha_{0} p}{(p-1) c_{0}}}\left(-h^{\prime}\right)^{p} Y_{1}^{-1+\alpha_{1}}\left(\frac{(p-1) c_{0}}{p}+\frac{\eta}{p}\right)^{p} \mathrm{~d} x
\end{aligned}
$$

where $\eta=-\alpha_{0}+\left(1-\alpha_{1}\right) Y_{1}$. Since $\eta$ is small compared to $(p-1) c_{0} / p$, we may use Taylor's expansion to obtain

$$
\begin{aligned}
\left(\frac{(p-1) c_{0}}{p}+\frac{\eta}{p}\right)^{p} \leq\left(\frac{(p-1) c_{0}}{p}\right)^{p}+\left(\frac{(p-1) c_{0}}{p}\right)^{p-1} \eta & \\
& +\frac{p-1}{2 p}\left(\frac{(p-1) c_{0}}{p}\right)^{p-2} \eta^{2}+C \eta^{3}
\end{aligned}
$$

Using this inequality we can obtain

$$
I_{1} \leq I_{10}+I_{11}+I_{12}+I_{13}
$$

where

$$
\begin{aligned}
I_{10} & =\int_{\Omega} \theta^{p} \phi h^{-\frac{\alpha_{0} p}{(p-1) c_{0}}}\left(-h^{\prime}\right)^{p} Y_{1}^{-1+\alpha_{1}} \mathrm{~d} x=\int_{\Omega} \theta^{p} \psi h^{p-\frac{\alpha_{0} p}{(p-1) c_{0}}} Y_{1}^{-1+\alpha_{1}} \mathrm{~d} x \\
& =\int_{\Omega} \theta^{p} \psi|w|^{p} \mathrm{~d} x=\int_{\Omega} \psi|u|^{p} \mathrm{~d} x \\
I_{12} & =\frac{p}{2(p-1) c_{0}^{2}} \int_{\Omega} \theta^{p} \phi h^{-\frac{\alpha_{0} p}{(p-1) c_{0}}}\left(-h^{\prime}\right)^{p} Y_{1}^{-1+\alpha_{1}} \eta^{2} \mathrm{~d} x
\end{aligned}
$$


We shall prove that

$$
I_{11}, I_{13}=O(1) \quad \text { uniformly in } \alpha_{0}, \alpha_{1} .
$$

Firstly,

$$
\begin{aligned}
I_{11}= & \frac{p}{(p-1) c_{0}}\left[-\alpha_{0} \int_{\Omega} \theta^{p} \phi\left(-h^{\prime}\right)^{p} h^{-\frac{\alpha_{0} p}{(p-1) c_{0}}} Y_{1}^{-1+\alpha_{1}} \mathrm{~d} x\right. \\
& \left.+\left(1-\alpha_{1}\right) \int_{\Omega} \theta^{p} \phi\left(-h^{\prime}\right)^{p} h^{-\frac{\alpha_{0} p}{(p-1) c_{0}}} Y_{1}^{\alpha_{1}} \mathrm{~d} x\right]+O(1) \\
= & \frac{p}{(p-1) c_{0}}\left(-\alpha_{0} A_{0}+\left(1-\alpha_{1}\right) \Gamma_{01}\right)+O(1)
\end{aligned}
$$

Next we estimate $I_{13}$.

$$
\begin{aligned}
I_{13} & \leq \alpha_{0}^{3} \int_{\Omega} \theta^{p} \phi\left(-h^{\prime}\right)^{p} h^{-\frac{\alpha_{0} p}{(p-1) c_{0}}} Y_{1}^{-1+\alpha_{1}} \mathrm{~d} x+C \int_{\Omega} \theta^{p} \phi\left(-h^{\prime}\right)^{p} h^{-\frac{\alpha_{0} p}{(p-1) c_{0}}} Y_{1}^{2+\alpha_{1}} \mathrm{~d} x \\
& =: I_{13}^{\prime}+I_{13}^{\prime \prime}
\end{aligned}
$$

Since

$$
Y_{1}^{-1}=\frac{p}{(p-1) c_{0}} \ln \frac{h(r)}{h(D)}
$$

we have

$$
\begin{gathered}
I_{13}^{\prime} \leq C \alpha_{0}^{3} \int_{0}^{\delta}\left(\int_{r}^{\infty}\left(\phi r^{k-1}\right)^{-1 /(p-1)} \mathrm{d} r\right)^{-1-\alpha_{0} / c_{0}}\left[\ln \frac{h(r)}{h(D)}\right]^{2} \\
\mathrm{~d}\left(\int_{r}^{\infty}\left(\phi r^{k-1}\right)^{-1 /(p-1)} \mathrm{d} r\right) \\
\leq C \alpha_{0}^{2} c_{0} \int_{0}^{\delta}\left[\ln \frac{h(r)}{h(D)}\right]^{2} \mathrm{~d}\left(\int_{r}^{\infty}\left(\phi r^{k-1}\right)^{-1 /(p-1)} \mathrm{d} r\right)^{-\alpha_{0} / c_{0}}
\end{gathered}
$$

Denote

$$
s=\left(\int_{d}^{\infty}\left(\phi r^{k-1}\right)^{-1 /(p-1)} \mathrm{d} r\right)^{-\alpha_{0} / c_{0}}
$$

then we have

$$
I_{13}^{\prime} \leq C \alpha_{0}^{2} \int_{0}^{\delta}\left[C-\frac{(p-1) c_{0}}{p \alpha_{0}} \ln s\right]^{2} \mathrm{~d} s \leq O(1)
$$

The same argument gives $I_{13}^{\prime \prime}=O(1)$ uniformly in $\alpha_{0}$ and $\alpha_{1}$. Hence, by (3.4), (3.6), (3.8), (3.9) and (3.11), we conclude that

$$
\int_{\Omega} \phi|\nabla u|^{p} \mathrm{~d} x-\int_{\Omega} \psi|u|^{p} \mathrm{~d} x \leq I_{12}+O(1)
$$


uniformly in $\alpha_{0}$ and $\alpha_{1}$.

Step 4. We proceed to estimate $I_{12}$ and complete the proof.

$$
\begin{aligned}
I_{12} & =\frac{p}{2(p-1) c_{0}^{2}} \int_{\Omega} \theta^{p} \phi h^{-\frac{\alpha_{0} p}{(p-1) c_{0}}}\left(-h^{\prime}\right)^{p} Y_{1}^{-1+\alpha_{1}}\left(\alpha_{0}^{2}+\left(1-\alpha_{1}\right)^{2} Y_{1}^{2}-2 \alpha_{0}\left(1-\alpha_{1}\right) Y_{1}\right) \mathrm{d} x \\
& =\frac{p}{2(p-1) c_{0}^{2}}\left(\alpha_{0}^{2} A_{0}-2 \alpha_{0}\left(1-\alpha_{1}\right) \Gamma_{01}+\left(1-\alpha_{1}\right)^{2} A_{1}\right) \\
& =\frac{p}{2(p-1) c_{0}^{2}} A_{1}+O(1)
\end{aligned}
$$

if $\alpha_{0}$ and $\alpha_{1}$ tend to 0 . Because

$$
\phi\left(-h^{\prime}\right)^{p} h^{-\frac{\alpha_{0} p}{(p-1) c_{0}}}=\left(c_{0} \int_{r}^{a}\left(\phi r^{N-1}\right)^{-1 /(p-1)} \mathrm{d} r\right)^{-\alpha_{0} / c_{0}-1} \cdot c_{0} \phi\left(\phi r^{N-1}\right)^{-1 /(p-1)}
$$

we have

$$
\begin{gathered}
A_{1} \geq C \int_{0}^{\delta / 2}\left(\int_{r}^{a}\left(\phi r^{N-1}\right)^{-1 /(p-1)} \mathrm{d} r\right)^{-\alpha_{0} / c_{0}-1} \cdot c_{0}\left(\phi r^{N-1}\right)^{-1 /(p-1)} h_{1}^{-1-\alpha_{0}} \mathrm{~d} r \\
\left.\left.\geq\left. C \frac{\left(\int_{r}^{a}\left(\phi r^{N-1}\right)^{-1 /(p-1)} \mathrm{d} r\right)^{-\alpha_{0} / c_{0}}}{-\alpha_{0} / c_{0}}\right|_{0} ^{\delta / 2}\right)^{-\alpha_{0} / c_{0}}-\left(\int_{\delta / 2}^{a}\left(\phi r^{N-1}\right)^{-1 /(p-1)} \mathrm{d} r\right)^{-\alpha_{0} / c_{0}}\right] \rightarrow \infty
\end{gathered}
$$

as $\alpha_{0}$ tends to 0 . Since

$$
\begin{aligned}
\int_{\Omega} \psi h_{1}^{-2}|u|^{p} \mathrm{~d} x & =\int_{\Omega} \phi\left(-\frac{h^{\prime}}{h}\right)^{p} h_{1}^{-2} \theta^{p} h^{p-\frac{\alpha_{0} p}{(p-1) c_{0}}} h_{1}^{1-\alpha_{1}} \mathrm{~d} x \\
& =\int_{\Omega} \theta^{p} \phi\left(-h^{\prime}\right)^{p} h^{-\frac{\alpha_{0} p}{(p-1) c_{0}}} h_{1}^{-1-\alpha_{1}} \mathrm{~d} x=A_{1}
\end{aligned}
$$

by (3.12) and (3.13), we have

$$
\frac{\int_{\Omega}\left(\phi|\nabla u|^{p}-\psi|u|^{p}\right) \mathrm{d} x}{\int_{\Omega} \psi h_{1}^{-2}|u|^{p} \mathrm{~d} x} \leq \frac{\frac{p}{2(p-1) c_{0}^{2}} A_{1}+O(1)}{A_{1}} \rightarrow \frac{p}{2(p-1) c_{0}^{2}}
$$

as $\alpha_{0}$ tends to 0 . This completes the proof.

The proof of Theorem 1.3. i) Assume $\left(\mathrm{A}_{1}\right)$ holds. Consider the case of $p \geq 2$. 
Let $u \in C_{0}^{\infty}(\Omega)$ and set $v=u(x) / h(r)$. Then by Lemma $2.1(2.2)$, we have

$$
\begin{aligned}
\int_{\Omega} \phi|\nabla u|^{p} \mathrm{~d} x & =\int_{\Omega} \phi\left|v h^{\prime} \frac{x}{|x|}+h \nabla v\right|^{p} \mathrm{~d} x \\
& \geq \int_{\Omega} \phi|v|^{p}\left|h^{\prime}\right|^{p} \mathrm{~d} x-p \int_{\Omega} \phi\left|v h^{\prime}\right|^{p-2}\left\langle v h^{\prime} \frac{x}{|x|}, h \nabla v\right\rangle \mathrm{d} x \\
& +c(p) \int_{\Omega} \phi h^{p}|\nabla v|^{p} \mathrm{~d} x
\end{aligned}
$$

Note that

$$
\int_{\Omega} \phi|v|^{p}\left|h^{\prime}\right|^{p} \mathrm{~d} x=\int_{\Omega} \psi|u|^{p} \mathrm{~d} x
$$

and for any $\epsilon>0$, by Lemma $2.2,(3.7)$ and $\left(\mathrm{A}_{1}\right)$ (or $\left(\mathrm{A}_{2}\right)$ ), we have

$$
\begin{aligned}
& -\int_{\Omega \backslash B_{\epsilon}(0)} \phi\left|v h^{\prime}\right|^{p-2} \mathrm{~d} x \\
= & \int_{\Omega \backslash B_{\epsilon}(0)} \phi h\left(-h^{\prime}\right)^{p-1}\left\langle\frac{x}{|x|}, h \nabla|v|^{p}\right\rangle \mathrm{d} x \\
= & \int_{\partial B_{\epsilon}(0)} \phi\left(-h^{\prime}\right)^{p-1} h|v|^{p} \mathrm{~d} S-\int_{\Omega \backslash B_{\epsilon}(0)}|v|^{p} \operatorname{div}\left(\phi h\left(-h^{\prime}\right)^{p-2} \nabla h\right) \\
= & \int_{\partial B_{\epsilon}(0)} \phi\left(-h^{\prime}\right)^{p-1} h|v|^{p} \mathrm{~d} S \rightarrow 0
\end{aligned}
$$

as $\epsilon \rightarrow 0$. Hence, we obtain

$$
I_{1, \phi}(u)=\int_{\Omega}\left(\phi|\nabla u|^{p}-\psi|u|^{p}\right) \mathrm{d} x \geq c(p) \int_{\Omega} \phi h^{p}|\nabla v|^{p} \mathrm{~d} x
$$

Taking $C_{1}>0$ such that $C_{1} h_{1}^{-2} \leq c(p)$, it follows from (2.2) of Lemma 2.1 that

$$
\begin{gathered}
c(p) \int_{\Omega} \phi h^{p}|\nabla v|^{p} \mathrm{~d} x \geq C_{1} \int_{\Omega} \phi h^{p} h_{1}^{-2}|\nabla v|^{p} \mathrm{~d} x \\
\geq C_{1} \int_{\Omega} \phi h_{1}^{-2}\left[\left|\frac{\nabla h_{1}}{h_{1}}\right||u|^{p}-p\left|\frac{\nabla h}{h} u\right|^{p-1}|\nabla u|+c(p)|\nabla u|^{p}\right] \mathrm{d} x \\
\geq C_{1} \int_{\Omega} \phi h_{1}^{-2}\left[(c(p)-\epsilon)|\nabla u|^{p}-\left((p-1) \epsilon^{-1 /(p-1)}-1\right)\left(-\frac{h^{\prime}}{h}\right)^{p}|u|^{p}\right] \mathrm{d} x
\end{gathered}
$$

Taking $\epsilon=c(p) / 2$, then by Theorem 1.2 , we obtain

$$
I_{1, \phi}(u) \geq C \int_{\Omega} \phi h_{1}^{-2}|\nabla u|^{p} \mathrm{~d} x
$$


Now let $1<p<2$. By using Lemma 2.1, (2.1) and arguments analogues to the case of $p \geq 2$, we have

$$
\begin{aligned}
\int_{\Omega}\left(\phi|\nabla u|^{p}-\psi|u|^{p}\right) \mathrm{d} x \geq c(p) \int_{\Omega} \frac{\phi\left|\nabla u-\frac{\nabla h}{h} u\right|^{p}}{\left(|\nabla u|+|u|\left|\frac{h^{\prime}}{h}\right|\right)^{2-p}} \mathrm{~d} x & \\
& \geq c(p) \int_{\Omega} \frac{\phi h_{1}^{-2}\left|\nabla u-\frac{\nabla h}{h} u\right|^{2}}{\left(|\nabla u|+|u|\left|\frac{h^{\prime}}{h}\right|\right)^{2-p}} \mathrm{~d} x
\end{aligned}
$$

By Hölder's inequality and Corollary 2.4, we have

$$
\begin{aligned}
\int_{\Omega} \phi h_{1}^{-2}\left|\nabla u-\frac{\nabla h}{h} u\right|^{p} \mathrm{~d} x & \leq\left(\int_{\Omega} \frac{\phi h_{1}^{-2}\left|\nabla u-\frac{\nabla h}{h} u\right|^{2}}{\left(|\nabla u|+|u|\left|\frac{h^{\prime}}{h}\right|\right)^{2-p}} \mathrm{~d} x\right)^{p / 2} \\
& \left(\int_{\Omega} \phi h_{1}^{-2}\left(|\nabla u|+\left|\frac{h^{\prime}}{h}\right||u|\right)^{p} \mathrm{~d} x\right)^{1-p / 2} \\
& \leq C\left(I_{1, \phi}(u)\right)^{p / 2}\left(\int_{\Omega} \phi h_{1}^{-2}|\nabla u|^{p} \mathrm{~d} x\right)^{1-p / 2}
\end{aligned}
$$

Note that

$$
\begin{aligned}
\int_{\Omega} \phi h_{1}^{-2}|\nabla u|^{p} \mathrm{~d} x & \leq C\left(\int_{\Omega} \phi h_{1}^{-2}\left|\nabla u-\frac{\nabla h}{h} u\right|^{p} \mathrm{~d} x+\int_{\Omega} \phi h_{1}^{-2}\left|\frac{\nabla h}{h}\right||u|^{p} \mathrm{~d} x\right) \\
& \leq C\left(I_{1, \phi}(u)\right)^{p / 2}\left(\int_{\Omega} \phi h_{1}^{-2}|\nabla u|^{p} \mathrm{~d} x\right)^{1-p / 2}+\int_{\Omega} \psi h_{1}^{-2}|u|^{p} \mathrm{~d} x \\
& \leq C\left(I_{1, \phi}(u)\right)^{p / 2}\left(\int_{\Omega} \phi h_{1}^{-2}|\nabla u|^{p} \mathrm{~d} x\right)^{1-p / 2}+I_{1, \phi}(u)
\end{aligned}
$$

By Young's inequality, we obtain

$$
\int_{\Omega} \phi h_{1}^{-2}|\nabla u|^{p} \mathrm{~d} x \leq C I_{1, \phi}(u)
$$

One can prove the result for the case of $\left(\mathrm{A}_{2}\right)$ by the analogues argument.

ii) Let $w$ and $u$ be as those defined in the proof of Theorem 1.2 (2), and let $p>2$. First, it follows from (3.12) and (3.13) that

$$
\int_{\Omega} \phi|\nabla u|^{p} \mathrm{~d} x-\int_{\Omega} \psi|u|^{p} \mathrm{~d} x \leq \frac{p}{2(p-1) c_{0}^{2}} A_{1}+O(1)
$$

By (2.2) we have

$$
|\nabla u|^{p}=|\theta \nabla w+w \nabla \theta|^{p} \geq \theta^{p}|\nabla w|^{p}-p \theta^{p-1}|\nabla w|^{p-1}|\nabla \theta| w+c(p)|w|^{p}|\nabla \theta|^{p}
$$


Hence

$$
\begin{aligned}
& \int_{\Omega} \phi h_{1}^{-2} g(x)|\nabla u|^{p} \mathrm{~d} x \geq \min _{x \in B_{\delta}(0)} g(x) \int_{B_{\delta}(0)} \phi h_{1}^{-2} \theta^{p}|\nabla w|^{p} \mathrm{~d} x+ \\
& c(p) \int_{B_{\delta}(0)} \phi h_{1}^{-2} g(x)|w|^{p}|\nabla \theta|^{p} \mathrm{~d} x-p \int_{B_{\delta}(0)} \phi h_{1}^{-2} g(x) \theta^{p-1}|\nabla \theta||\nabla w|^{p-1}|w| \mathrm{d} x
\end{aligned}
$$

Analogues to the argument of Step 3 for the proof of Theorem 1.2 (2), we obtain

$$
\int_{B_{\delta}(0)} \phi h_{1}^{-2} \theta^{p}|\nabla w|^{p} \mathrm{~d} x=\int_{B_{\delta}(0)} \phi h_{1}^{-2} \theta^{p} h^{-\frac{\alpha_{0} p}{(p-1) c_{0}}}\left(-h^{\prime}\right)^{p} Y_{1}^{-1+\alpha_{1}}\left(\frac{(p-1) c_{0}}{p}+\frac{\eta}{p}\right)^{p} \mathrm{~d} x
$$

where $\eta=-\alpha_{0}+\left(1-\alpha_{1}\right) Y_{1}$. Because of $p>2$, we have

$$
\begin{aligned}
\left(\frac{(p-1) c_{0}}{p}+\frac{\eta}{p}\right)^{p} & =\left(\frac{(p-1) c_{0}-\alpha_{0}}{p}+\frac{\eta+\alpha_{0}}{p}\right)^{p} \\
& \geq\left(\frac{(p-1) c_{0}-\alpha_{0}}{p}\right)^{p}+\left(\frac{(p-1) c_{0}-\alpha_{0}}{p}\right)^{p-1}\left(\eta+\alpha_{0}\right)
\end{aligned}
$$

Hence

$$
\begin{gathered}
\int_{B_{\delta}(0)} \phi h_{1}^{-2} \theta^{p}|\nabla w|^{p} \mathrm{~d} x \geq\left(\frac{(p-1) c_{0}-\alpha_{0}}{p}\right)^{p} \int_{B_{\delta}(0)} \phi \theta^{p} h^{-\frac{\alpha_{0} p}{(p-1) c_{0}}}\left(-h^{\prime}\right)^{p} Y_{1}^{1+\alpha_{1}} \mathrm{~d} x \\
+\left(\frac{(p-1) c_{0}-\alpha_{0}}{p}\right)^{p-1}\left(1-\alpha_{1}\right) \int_{B_{\delta}(0)} \phi \theta^{p} h^{-\frac{\alpha_{0} p}{(p-1) c_{0}}\left(-h^{\prime}\right)^{p} Y_{1}^{2+\alpha_{1}} \mathrm{~d} x} \\
=: J_{1}+J_{2}
\end{gathered}
$$

By Step 3 of the proof of Theorem 1.2 (2) we know

$$
J_{1}=\left(\frac{(p-1) c_{0}-\alpha_{0}}{p}\right)^{p} A_{1}, \quad J_{2}=O(1)
$$

if $\alpha_{0}, \alpha_{1}$ tend to 0 . Next, we will estimate

$$
J_{3}:=\int_{B_{\delta}(0)} \phi h_{1}^{-2} g(x) \theta^{p-1}|\nabla \theta||\nabla w|^{p-1}|w| \mathrm{d} x
$$

In fact,

$$
\begin{aligned}
& J_{3} \leq C \int_{B_{\delta}(0)} g(x) \phi h_{1}^{-2} h^{1-\frac{\alpha_{0} p}{(p-1) c_{0}}}\left(-h^{\prime}\right)^{p-1} Y_{1}^{-1+\alpha_{1}}\left[(p-1) c_{0}-\alpha_{0}+\left(1-\alpha_{1}\right) Y_{1}\right]^{p-1} \mathrm{~d} x \\
\leq & \int_{B_{\delta}(0)} g(x) \phi h_{1}^{-2} h^{1-\frac{\alpha_{0} p}{(p-1) c_{0}}}\left(-h^{\prime}\right)^{p-1}\left[\left((p-1) c_{0}-\alpha_{0}\right)^{p-1} Y_{1}^{1+\alpha_{1}}+\left(1-\alpha_{1}\right)^{p-1} Y_{1}^{p+\alpha_{1}}\right] \mathrm{d} x
\end{aligned}
$$


It follows from (3.7) that

$$
J_{3} \leq \int_{0}^{\delta} \widetilde{g}(r) h^{-\frac{\alpha_{0} p}{(p-1) c_{0}}}\left[\left((p-1) c_{0}-\alpha_{0}\right)^{p-1} Y_{1}^{1+\alpha_{1}}+\left(1-\alpha_{1}\right)^{p-1} Y_{1}^{p+\alpha_{1}}\right] \mathrm{d} r
$$

Set

$$
\widetilde{g}(r)=\frac{1}{N \omega_{N}} \int_{|\omega|=1} g(r \omega) \mathrm{d} \omega
$$

and we may assume

$$
\left|\widetilde{g}(r) h_{1}^{-1}(r)\right| \leq C
$$

Then we obtain

$$
J_{3} \leq C \int_{0}^{\delta} h^{-\frac{\alpha_{0} p}{(p-1) c_{0}}}\left[\left((p-1) c_{0}-\alpha_{0}\right)^{p-1} Y_{1}^{\alpha_{1}}+\left(1-\alpha_{1}\right)^{p-1} Y_{1}^{p-1+\alpha_{1}}\right] \mathrm{d} r \leq C
$$

Hence

$$
\frac{\int_{\Omega}\left(\phi|\nabla u|^{p}-\psi|u|^{p}\right) \mathrm{d} x}{\int_{\Omega} \phi h_{1}^{-2} g(x)|\nabla u|^{p} \mathrm{~d} x} \leq \frac{\frac{p}{2(p-1) c_{0}^{2}} A_{1}+O(1)}{\min _{x \in B_{\delta}(0)} g(x)\left(\frac{(p-1) c_{0}-\alpha_{0}}{p}\right)^{p} A_{1}+O(1)} \rightarrow 0
$$

as $\delta \rightarrow 0$ since $A_{1} \rightarrow \infty$ as $\alpha_{0}, \alpha_{1} \rightarrow 0$ and $g(x) \rightarrow \infty$ as $x \rightarrow 0$.

We can prove our result for the case of $1<p<2$ by the similar argument.

\section{References}

[1] B. Abdellaoui, E. Colorado and I. Peral, Some improved Caffarelli-KohnNirenberg inequalities, Calc. Var. Partial Differential Equations 23 (2005), no. $3,327-345$

[2] G. Barbatis, S. Filippas and A. Tertikas, A unified approach to improved $L^{p}$ Hardy inequalities with best constants, Trans. Amer. Math. Soc., 356(2004), 2169-2196

[3] Brezis H, Vázquez, J L. Blow-up solutions of some nonlinear elliptic problems. Rev. Mat. Univ. Complut. Madrid, 1997, 10(2): 443-469

[4] L. Cafferelli, R. Kohn and L. Nirenberg, First order interpolation inequality with weights, Compositio Math., 53(1984), 259-275 
[5] Z. H. Chen and Y. T. Shen, Hardy-Sobolev inequalities with general weights and remainder terms, to appear in Acta Math. Sci.

[6] F. Catrina and Z. Q. Wang, On the Caffarelli-Kohn-Nirenberg inequlities: Sharp constants existence (and nonexistence), and symmetry of extremal functions, C. P. A. M., 54(2): 229-258, 2001

[7] Hardy G H. Note on a theorem of Hilbert. Math Z, 1920, 6(3-4): 314-317

[8] J. Leray, Étude de diverses équations intégrales non linéaires et de quelques problèmes que pose l'hydrodynamique, J. Math. Pures Appl., 12(1933), 1-82 (French)

[9] Shen, Y. T., Weak solutions of elliptic equations of second order with singular coefficients, Advances in Mathematics, 1964, 7:321-327, in Chinese

[10] Shen Y. T., The Dirichlet problem for degenerate or singular elliptic equation of high order, J. China Univ. Sci. Tech., 10 (1980), no. 2, 15-25, in Chinese

[11] Shen Y. T. and Gu Y. G., Poincaré inequalities on unbounded domains and strong nonlinear variations. Chinese Sci. Bull., 27 (1982), no. 10, 1033-1036

[12] Shen, Y. T., Guo, X. K., Weighted Poincaré inequalities on unbounded domains and nonlinear elliptic boundary value problems, Acta. Math. Soc., $1984,4: 265-274$

[13] Shen Y. T. and Chen Z. H., General Hardy inequalities with optimal constants and remainder terms, J. Inequal. Appl., 2005:3207-219, 2005

[14] Shen Y. T. and Chen Z. H., Sobolev-Hardy space with general weight, J. Math. Anal. Appl., 320(2) (2006), 675-690

[15] Shen Y. T. and Yao Y. X., Nonlinear elliptic equations with critical potential and critical parameter, Proc. Royal Soc. Edinb., 136A, 1-11, 2006

[16] J. L. Vazquez and E. Zuazua, The Hardy inequality and the asymptotic behavior of the heat equation with an inverse-square potential, J. Funct. Anal., 173(2000), 103-153 
[17] Z. Q. Wang and M. Willem, Caffarelli-Kohn-Nirenberg inequalities with remainder terms. J. Funct. Anal., 203 (2003), no. 2, 550-568

Yaotian Shen

Department of Mathematics

South China University of Technology

Guangzhou 510640, China

E-mail: maytshen@scut.edu.cn

Zhihui Chen

Department of Mathematics

South China University of Technology

Guangzhou 510640, China

E-mail: mazhchen@scut.edu.cn 